\title{
Rapid and sensitive hormonal profiling of complex plant samples by liquid chromatography coupled to electrospray ionization tandem mass spectrometry
}

\author{
Maren Müller and Sergi Munné-Bosch*
}

\begin{abstract}
Background: Plant hormones play a pivotal role in several physiological processes during a plant's life cycle, from germination to senescence, and the determination of endogenous concentrations of hormones is essential to elucidate the role of a particular hormone in any physiological process. Availability of a sensitive and rapid method to quantify multiple classes of hormones simultaneously will greatly facilitate the investigation of signaling networks in controlling specific developmental pathways and physiological responses. Due to the presence of hormones at very low concentrations in plant tissues $\left(10^{-9} \mathrm{M}\right.$ to $\left.10^{-6} \mathrm{M}\right)$ and their different chemistries, the development of a high-throughput and comprehensive method for the determination of hormones is challenging.

Results: The present work reports a rapid, specific and sensitive method using ultrahigh-performance liquid chromatography coupled to electrospray ionization tandem spectrometry (UPLC/ESI-MS/MS) to analyze quantitatively the major hormones found in plant tissues within six minutes, including auxins, cytokinins, gibberellins, abscisic acid, 1-amino-cyclopropane-1-carboxyic acid (the ethylene precursor), jasmonic acid and salicylic acid. Sample preparation, extraction procedures and UPLC-MS/MS conditions were optimized for the determination of all plant hormones and are summarized in a schematic extraction diagram for the analysis of small amounts of plant material without time-consuming additional steps such as purification, sample drying or resuspension.

Conclusions: This new method is applicable to the analysis of dynamic changes in endogenous concentrations of hormones to study plant developmental processes or plant responses to biotic and abiotic stresses in complex tissues. An example is shown in which a hormone profiling is obtained from leaves of plants exposed to salt stress in the aromatic plant, Rosmarinus officinalis.
\end{abstract}

Keywords: UPLC/ESI-MS/MS, Phytohormones, Auxins, Abscisic acid, Cytokinins, Gibberellins, Salicylic acid, Jasmonic acid, 1-amino-cyclopropane-1-carboxyic acid, Rosmarinus officinalis

\section{Background}

Hormones play a pivotal role in most physiological processes in plants. These structurally diverse compounds that act usually at nanomolar levels include five groups of the so-called "classic" hormones, comprising auxins, cytokinins, gibberellins (GA), abscisic acid (ABA) and ethylene, and several other plant growth regulators,

\footnotetext{
* Correspondence: smunne@ub.edu

Departament de Biologia Vegetal, Facultat de Biologia, Universitat de Barcelona, Avinguda Diagonal, 645, E-08028 Barcelona, Spain
}

including jasmonates, salicylates, brassinosteroids, polyamines or the very recently discovered strigolactones, which fit several of the criteria to be considered hormones [1-3]. Furthermore, the list of plant hormones is expected to increase due to a better understanding of plant growth and development and stress responses, and the use of technological advances in analytical methods.

Recent studies support the contention that hormone actions build a signaling network and mutually regulate several signaling and metabolic systems, such as auxins

\section{(Ciomed Central}


and GAs in growth regulation [4], CKs, auxins, ABA and strigolactones in apical dominance $[2,5]$, auxins and brassinosteroids in cell expansion [6,7], ethylene and cytokinins in the inhibition of root and hypocotyl elongation [8], ethylene, ABA and GAs in some plant stress responses $[9,10]$, or SA, JA and auxin in plant responses to pathogens $[11,12]$ to name just a few of the reported hormonal interactions. Therefore, focusing on a single endogenous plant hormone to evaluate hormone-regulated physiological or developmental biological problems is not sufficient anymore [13].

In order to understand better the network regulation of hormone action influencing plant growth and development as well as the distribution of several hormones at the organ, cellular and sub-cellular levels, an ideal analytical method should provide a measure of multiple hormone concentrations (hormonal profiling) from a single experimental sample. Therefore several methods for the simultaneous quantification of multiple plant hormones using mass spectrometry with multiple reaction monitoring (MRM) have been developed recently. It has been reported a multiplex gas chromatographytandem mass spectrometry (GC-MS/MS) technique for the simultaneous analysis of SA, JA, IAA, ABA and OPDA in Arabidopsis thaliana [13]. However, GC-MS is limited to volatile compounds and as a result it is necessary to purify and derivatize hormones prior to analysis. Another potential downside in GC-MS procedures apart from the purification and derivatization is the use of high temperatures, which can degrade thermal labile compounds [14].

An alternative to GC-MS is liquid chromatography coupled to mass spectrometry (LC-MS). A high performance liquid chromatography-electrospray ionization tandem mass spectrometry (HPLC/ESI-MS/MS) method for the simultaneous analysis of 15 plant hormones and metabolites from four different hormone classes (auxins, cytokinins, GAs and ABA) has been reported to analyze hormone regulation of thermodormancy of lettuce seeds [15]. Also, a HPLC/ESI-MS/ MS method to analyze seven major classes of plant hormones including auxins, cytokinins, GAs, ABA, jasmonates, brassinosteriods and SA in Arabidopsis thaliana has been developed [1]. Furthermore, an ultrahigh-performance liquid chromatography electrospray ionization tandem mass spectrometry (UPLC/ ESI-MS/MS) technique to analyze cytokinins, auxins, ABA and GAs in rice has been described [16]. To improve the detection limit of the negatively charged compounds they derivatized auxin, ABA and GAs with bromocholin and analyzed all compounds in the positive ion mode. However, at present this method is limited and cannot target other plant hormones such as JA and SA.
Plant hormones are structurally diverse compounds with diverse physiochemical properties. The question as to whether all plant hormones can be extracted equally well has not yet been answered. The choice of extraction methods depends not only on the target analysts but also on the matrix of the analyzed tissues. The requirements on the extraction method increase with the complexity of the sample matrix. In the literature diverse extraction solvents such as methanol, methanolwater mixtures, isopropanol, or isopropanol-water mixtures have been used with one or two extraction steps [14,15,17-19]. In addition, time-consuming multiple steps of sample preparation procedures, including sample purification, drying of sample under $\mathrm{N}_{2}$ and re-suspension of the residues have been reported for plant hormone extraction $[14,20]$ which may increase the risk of hormone loss. However, the application of internal standards can provide corrections for hormone loss during sample preparation and chromatographic separation.

Here we developed a new method which allows to analyze dynamic changes in endogenous concentrations of major plant hormones and to study plant development processes or plant responses to biotic and abiotic stresses in complex sample matrices. An example is shown in which rosemary (Rosmarinus officinalis), an aromatic Mediterranean perennial shrub rich in secondary metabolites and epicuticular waxes, was exposed to salt stress. Soil salinity is one of the most serious environmental threats for plant survival and affects many undesirable changes in plants such as hyperionic and hyperosmotic effects, increase in reactive oxygen species and metabolic toxicity. These changes lead to growth reduction, changes in biomass allocation and phenology, leaf senescence, and finally to plant death [21-23]. It has been shown that senescence induced by salinity follows at least in part similar physiological events as droughtinduced senescence [24]. Plant hormones such as ABA, ethylene and cytokinins are involved in different plant strategies to overcome the damaging effects of salinity, however, the complex hormonal response is only partly known [25,26]. The present work reports a sensitive and rapid method to quantify 17 plant hormones from seven plant classes including auxins, cytokinins, GAs, ABA, ACC (the ethylene precursor), SA and JA in complex tissues using ultra-performance liquid chromatography mass spectrometry (UPLC/ESI-MS/MS) with multiple reaction monitoring (MRM). This method allows obtaining a hormonal profiling in $6 \mathrm{~min}$. Sample preparation, extraction procedures and UPLC-MS/MS conditions were optimized.

\section{Results and discussion}

Of the 17 endogenous plant hormones investigated, $\mathrm{Z}$, DHZ, 2-IP, IAA, ABA, JA, SA, ACC, GA $4, \mathrm{GA}_{9}, \mathrm{GA}_{24}$ 
were detected in rosemary leaves, whereas $\mathrm{ZR}, \mathrm{DHZR}$, IPA, $\mathrm{GA}_{1}, \mathrm{GA}_{19}$, and $\mathrm{GA}_{20}$ concentrations were under the limit of detection. However, the internal standards $\mathrm{d}_{4}-\mathrm{SA}, \mathrm{d}_{6}-\mathrm{ABA}, \mathrm{d}_{5}-\mathrm{JA}, \mathrm{d}_{5}-\mathrm{IAA}, \mathrm{d}_{2}-\mathrm{GA}_{1}, \mathrm{~d}_{2}-\mathrm{GA}_{4}, \mathrm{~d}_{2}-\mathrm{GA}_{9}$, $\mathrm{d}_{2}-\mathrm{GA}_{19}, \mathrm{~d}_{2}-\mathrm{GA}_{20}, \mathrm{~d}_{2}-\mathrm{GA}_{24}, \mathrm{~d}_{4}-\mathrm{ACC}, \mathrm{d}_{6}-2 \mathrm{iP}, \mathrm{d}_{6}-\mathrm{IPA}, \mathrm{d}_{5}-$ $Z$ and $d_{5}-Z R$ were detected in all rosemary leaf extracts $\left(d_{5}-Z\right.$ and $d_{5}-Z R$ were used as internal standards for $Z$, DHZ and ZR, DHZR).

\section{Extraction solvents}

The extraction of plant hormones will critically determine the quality of the results obtained. Therefore the choice of the extraction solvent is very important, however, it is also challenging by the structurally diversity of plant hormones. Previously reported methods for plant hormone extraction used predominately methanol, methanol mixtures or isopropanol. Four classes of plant hormones including auxins, cytokinins, ABA and gibberellins were extracted using isopropanol:glacial acetic acid (99:1; v/v) [15]. Methanol:water:acetic acid (10:79:1) was used to extract ABA, SA and JA [27]. In other studies methanol:water:formic acid $(75: 20.5)$ was used to extract cytokinins, IAA and ABA $[17,19]$. We tested methanol:glacial acetic acid, 99:1 (v/v), isopropanol:glacial acetic acid, 99:1 (v/v) and different methanol:isopropanol:glacial acetic acid mixtures, 80:19:1; 60:39:1; 40:59:1; 20:79:1 (v/v/v). Thirty-five $100 \mathrm{mg}$ samples of frozen rosemary leaves were extracted with 7 different extraction solvents including 5 replicates after incorporation of deuterated labeled plant hormones as internal standards.

Due to the structurally diversity of the 17 plant hormones a solvent alone was not able to extract all plant hormones equally well (Figure 1). Whereas ABA, SA, JA, GAs and IAA showed higher yields using solvents with higher concentrations of isopropanol than methanol, opposite results were found for cytokinins and ACC. In general it could be observed that methanol:isopropanol mixtures are favorable to extract plant hormones compared to $100 \%$ methanol or $100 \%$ isopropanol except for ACC. The results suggested that the choice of the extraction solvent depends on which plant hormones are of more interest to investigate. For the analysis of ABA, SA, JA, IAA and GAs methanol:

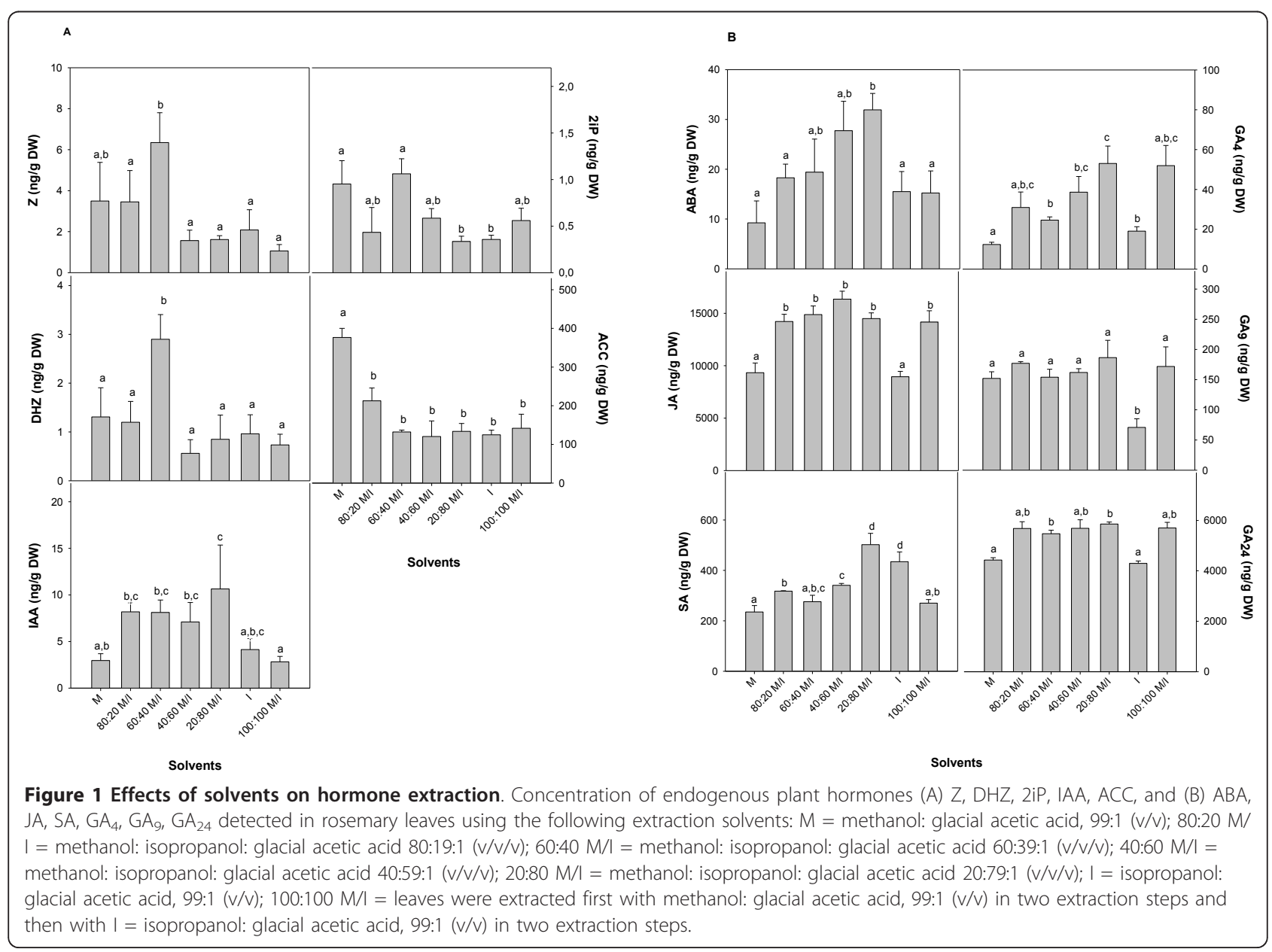


isopropanol:glacial acetic acid, 20:79:1 (v/v/v) is preferred, for cytokinins methanol:isopropanol:glacial acetic acid, 60:39:1 (v/v/v) and for ACC methanol:glacial acetic acid, 99:1 (v/v). Therefore the results indicate that plant hormones including amines (cytokinins and ACC) were dissolved preferentially in the more polar solvent whereas plant hormones including a carboxyl group (ABA, JA, SA, IAA, GAs) were dissolved in the less polar solvent. For the following experiments plant material was analyzed using the less polar extraction solvent methanol:isopropanol:glacial acetic acid, 20:79:1 (v/v/v) which is favorable to analyze ABA, SA, JA, GAs, and IAA. Recoveries about $>80 \%$ were found for ABA, SA, $\mathrm{GA}_{4}, \mathrm{GA}_{9}$, IAA; $77 \%$ for $\mathrm{d}_{2}-\mathrm{GA}_{24}$; between 62 and $50 \%$ for JA, $2 \mathrm{iP}, \mathrm{ACC}$; and $35 \%$ for $\mathrm{Z}$.

\section{Fresh or dried leaf material}

Little is known whether freeze drying (compared to fresh plant material) adversely affects plant hormone contents. A 25\% decrease was observed for SA and JA yields from freeze dried compared to fresh leaf material of Arabidopsis [27]. A decrease of $50 \%$ in SA but no change in JA levels of freeze dried material from cucumber compared to those from the equivalent amount of fresh tissue was measured [20]. Here plant hormone contents from fresh frozen and freeze dried leaf material of rosemary were compared. Leaves were collected and immediately frozen in liquid nitrogen. Ten fresh weight (FW) and ten freeze dried samples (DW) were then extracted after the addition of internal standards. Additional File 1 shows no significant differences in plant hormone contents comparing fresh frozen and freeze dried plant material, except for $\mathrm{GA}_{9}$, which showed significant higher contents in fresh samples.

\section{Extraction steps}

Undoubtedly, the requirements on the extraction methods increase with the complexity of the sample matrix. Rosemary leaves represent a complex matrix including essential oils, tannins, flavonoids, diterpenes, saponins, epicuticular waxes and resin. Five $100 \mathrm{mg}$ samples (fresh weight) were extracted five times after including internal standards. Each supernatant was immediately dried under nitrogen stream, re-suspended and injected to LC-MS. Additional File 2 shows clear differences regarding the necessary extraction steps for endogenous plant hormones. DHZ was only detectable in the first three extractions; $2 \mathrm{iP}, \mathrm{JA}$, and $\mathrm{GA}_{9}$ in four extractions; and $\mathrm{Z}$, $\mathrm{IAA}, \mathrm{ACC}, \mathrm{ABA}, \mathrm{SA}, \mathrm{GA}_{4}$ and $\mathrm{GA}_{24}$ in five extractions.

\section{Extract drying}

The concentration of sample extract by drying of samples under $\mathrm{N}_{2}$ and re-suspension of the residues is widespread in the literature $[14,15]$. However, each manipulation runs the risk of plant hormone loss apart from being time consuming. Twenty 25-50 mg samples (FW) were extracted after the addition of internal standards with $200 \mu \mathrm{l}$ of solvent extract (methanol: isopropanol: glacial acetic acid, 20:79:1, v/v) using ultra sonication. After centrifugation $\left(10,000 \mathrm{rpm}\right.$ for $15 \mathrm{~min}$ at $\left.4^{\circ} \mathrm{C}\right)$, the supernatant was collected and the pellet was re-extracted twice with $100 \mu \mathrm{l}$ of extraction solvent. The supernatants were combined and divided. One half was immediately injected and the other one was completely dried under nitrogen stream and re-suspended before being injected. For ABA, ACC, JA, DHZ, IAA, GA $, \mathrm{GA}_{9}, \mathrm{GA}_{24}$ and for $2 \mathrm{iP}$ and SA a significant loss about $70 \%$ and $50 \%$, respectively, could be observed for the dried extract compared to the immediately injected extract (Figure 2). Only Z showed higher levels for the dried and re-suspended extract. The high loss of plant hormones during the drying process indicates that sample extractions should immediately be injected, however, sample weight and volume of the extraction solvent must be adjusted.

\section{Quality parameters}

In the optimum LC-MS/MS conditions calibration curves were created using solutions containing varying amounts of each unlabeled analyte compound and a known fixed amount of deuterium labeled internal standard. The obtained calibration curves showed linearity of correlation coefficients $\left(R^{2}\right)$ in the concentration range selected between 0.996 and 0.999 for the different analysts.

To test the reproducibility of the developed method sample extracts were repeatedly injected (five times). The results show good reproducibility of elution times (relative standard deviations, RSDs, < 0.73) and peak areas (RSDs, < 5.07) (Table 1) for each compound.

Sensitivity parameters are listed in Table 2 where the limit of detection (LOD) and limit of quantification (LOQ) based on a signal-to-noise ratio of $3: 1$ and 10:1, respectively, were calculated through the standard addition curves. LOD for the different plant hormones ranged from $0.07 \mathrm{ng} \mathrm{g}^{-1}$ for DHZ to $12.1 \mathrm{ng} \mathrm{g}^{-1}$ for $\mathrm{GA}_{20}$, and LOQ ranged from $0.24 \mathrm{ng} \mathrm{g}^{-1}$ for DHZ to $40.33 \mathrm{ng}$ $\mathrm{g}^{-1}$ for $\mathrm{GA}_{20}$ in fresh rosemary leaves. To achieve a fully quantitative determination of plant hormones in plant tissues the necessary plant material has also to be taken into account. The minimum detectable amounts in samples analyzed were calculated regarding the limit of detection for each detectable plant hormone. The data shows that less than $0.1 \mathrm{mg}(\mathrm{FW})$ of leaf tissue is sufficient to determine ACC, SA, JA, GA $\mathrm{GA}_{24}$, while the analysis of $\mathrm{Z}, \mathrm{ABA}, \mathrm{IAA}, \mathrm{GA}_{4}$ and $\mathrm{GA}_{9}$ require amounts between 3 and $9 \mathrm{mg}(\mathrm{FW})$ and $\mathrm{DHZ}$ and 2iP amounts between 10 and $25 \mathrm{mg}$ (FW) (Table 3 ). The capacity of the extraction method to analyze different amounts of leaf tissue was also tested. Twenty-five rosemary 


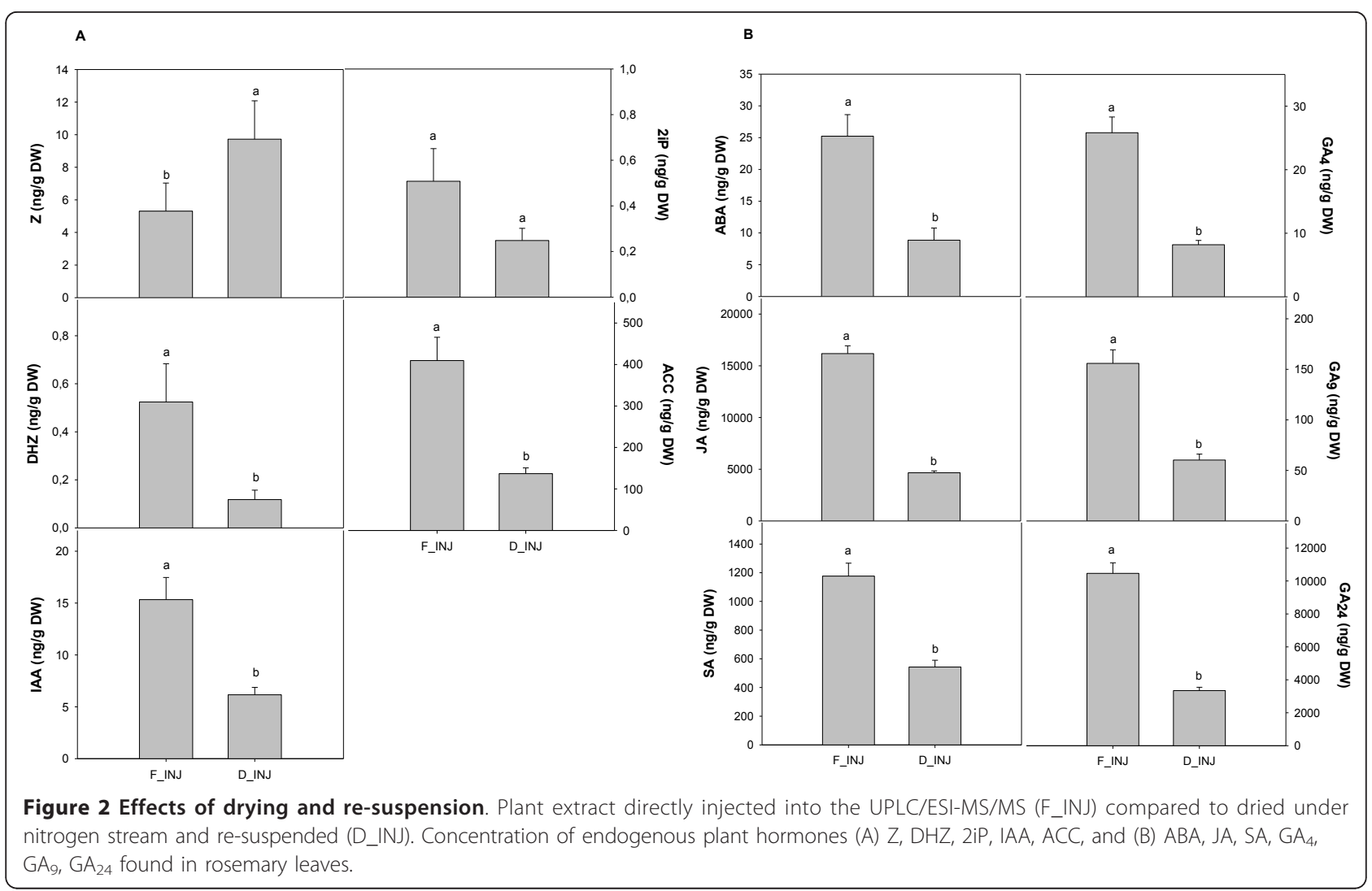

samples between 20 and $200 \mathrm{mg}$ (FW) were extracted after the addition of internal deuterium labeled plant hormones. Additional File 3 shows clearly that a linear relationship exists for all detected compounds of interest over the whole range of sample sizes $\left(R^{2}\right.$ values of 0.981 to 0.999 for all detected plant hormones).

\section{Sample stability}

It is quite important to develop a method that is not only simple, sensitive and rapid, but also a high

Table 1 Reproducibility of the developed LC/ESI-MS/MS method.

\begin{tabular}{|c|c|c|}
\hline Analyte/IS & $\begin{array}{l}\text { RRT }^{\mathrm{a}} \\
\mathrm{RSD}(\%)\end{array}$ & $\begin{array}{l}\text { RPA }^{\mathrm{b}} \\
\text { RSD (\%) }\end{array}$ \\
\hline $\mathrm{Z} / \mathrm{d}_{5}-\mathrm{Z}$ & 0.51 & 4.11 \\
\hline$\left|A A / d_{5}-\right| A A$ & 0.38 & 3.45 \\
\hline$A B A / d_{6}-A B A$ & 0.20 & 4.36 \\
\hline$J A / d_{5}-J A$ & 0.21 & 0.45 \\
\hline $\mathrm{SA} / \mathrm{d}_{4}-\mathrm{SA}$ & 0.21 & 0.47 \\
\hline$A C C / d_{4}-A C C$ & 0.73 & 2.78 \\
\hline $\mathrm{GA}_{4} / \mathrm{d}_{2}-\mathrm{GA}_{4}$ & 0.20 & 1.72 \\
\hline $\mathrm{GA}_{9} / \mathrm{d}_{2}-\mathrm{GA}_{9}$ & 0.00 & 4.52 \\
\hline $\mathrm{GA}_{24} / \mathrm{d}_{2}-\mathrm{GA}_{24}$ & 0.19 & 1.11 \\
\hline
\end{tabular}

a Relative retention time

${ }^{\mathrm{b}}$ Relative peak area throughput screening is desirable. To test the stability, analyzed sample extracts remained in the autosampler $\left(4^{\circ} \mathrm{C}\right)$ for $48 \mathrm{~h}$ and were then re-injected. Table 4 summarizes the ratio of plant hormones to internal standards at 0 and $48 \mathrm{~h}$. No significant degradation of samples was found over $48 \mathrm{~h}$ (relative retention time, RSD $<1.35$ and relative peak area, RDS $<9.47$ ), indicating that this method allows preparing and screening about 450 samples in 2 days.

\section{Hormonal profiling of rosemary leaves under salt stress}

Rosemary is a moderately salt-tolerant glycophyte $[28,29]$. Plants exposed to $200 \mathrm{mM} \mathrm{NaCl}$ during the experiment suffered salt stress indicated by a decrease of RWC and the $F_{\mathrm{v}} / F_{\mathrm{m}}$ ratio (Table 5 ). It has been shown that a number of plant hormones play pivotal roles to overcome damaging effects of salinity in plants, mainly the anti-stress defense compounds ABA, ethylene, SA and JA [24]. ABA regulates not only stomatal closure and hydraulic conductivity, but also root and shoot growth under salt stress conditions [30,31]. Ethylene has also been reported to be involved in saltinduced senescence [32,33]. It has been found increasing amounts of ACC, the precursor of ethylene, in tomato plants during salinity [22]. SA and JA have also been suggested to be involved in cellular signaling in plant 
Table 2 LOD and LOQ values.

\begin{tabular}{|c|c|c|}
\hline Analyte & $\begin{array}{c}\text { LOD }^{\mathrm{a}} \\
\text { (ng/g DW) }\end{array}$ & $\begin{array}{c}\mathrm{LOQ}^{\mathrm{b}} \\
(\mathrm{ng} / \mathrm{g} \mathrm{DW})\end{array}$ \\
\hline Z & 0.09 & 0.29 \\
\hline ZR & 4.20 & 13.99 \\
\hline $\mathrm{DHZ}$ & 0.07 & 0.24 \\
\hline DHZR & 0.21 & 0.69 \\
\hline $2 \mathrm{iP}$ & 0.16 & 0.52 \\
\hline IPA & 1.54 & 5.14 \\
\hline IAA & 0.48 & 1.59 \\
\hline$A B A$ & 0.31 & 1.04 \\
\hline JA & 1.24 & 4.13 \\
\hline SA & 0.21 & 0.70 \\
\hline $\mathrm{ACC}$ & 0.23 & 0.78 \\
\hline $\mathrm{GA}_{1}$ & 4.30 & 14.35 \\
\hline $\mathrm{GA}_{4}$ & 0.23 & 0.78 \\
\hline $\mathrm{GA}_{9}$ & 1.42 & 4.73 \\
\hline $\mathrm{GA}_{19}$ & 0.15 & 0.51 \\
\hline $\mathrm{GA}_{20}$ & 12.10 & 40.33 \\
\hline $\mathrm{GA}_{24}$ & 5.29 & 17.63 \\
\hline
\end{tabular}

${ }^{a}$ Limit of detection $(\mathrm{S} / \mathrm{N}=3)$

b Limit of quantification $(S / N=10)$

response to salinity [34-36]. Furthermore, essential regulators of plant growth, such as cytokinins, auxins and GAs are also sensitive to experience changes during salt stress responses, since this environmental constraint is known to inhibit leaf growth. Therefore, obtaining a hormone profile is essential to understand the delicate balance of regulators in plant responses to salinity, and the profiling obtained will obviously depend on the species examined, duration and magnitude of stress and specific conditions of study. Here we present the hormone profile of rosemary plants in response to salinity with a distinction in the response between young and old leaves (Figure 3). Results show that senescence in

Table 3 Requirements of plant material for the UPLC-MS/ MS analysis of endogenous plant hormones in Rosmarinus officinalis plants.

\begin{tabular}{lc}
\hline Analyte & $\begin{array}{c}\text { Minimum tissue requirement } \\
\text { mg (FW) }\end{array}$ \\
\hline $\mathbf{Z}$ & 4.54 \\
DHZ & 24.38 \\
2iP & 24.52 \\
IAA & 8.62 \\
ABA & 2.61 \\
JA & 0.02 \\
SA & 0.03 \\
ACC & 0.09 \\
$\mathrm{GA}_{\mathbf{4}}$ & 1.77 \\
$\mathrm{GA}_{\mathbf{9}}$ & 1.27 \\
$\mathrm{GA}_{\mathbf{2 4}}$ & 0.09 \\
\hline
\end{tabular}

Table 4 Plant hormones remain stable $48 \mathrm{~h}$ after extraction.

\begin{tabular}{|c|c|c|}
\hline Analyte/IS & $\begin{array}{c}\mathrm{RRT}^{\mathrm{a}} \\
\mathrm{RDS}(\%)\end{array}$ & $\begin{array}{c}\text { RPA }^{\mathrm{b}} \\
\text { RDS (\%) }\end{array}$ \\
\hline $\mathrm{Z} / \mathrm{d}_{5}-\mathrm{Z}$ & 0.76 & 9.47 \\
\hline$\left|A A / d_{5}-\right| A A$ & 0.43 & 8.96 \\
\hline$A B A / d_{6}-A B A$ & 0.25 & 5.05 \\
\hline$J A / d_{5}-J A$ & 0.26 & 1.72 \\
\hline $\mathrm{SA} / \mathrm{d}_{4}-\mathrm{SA}$ & 0.27 & 2.79 \\
\hline $\mathrm{ACC} / \mathrm{d}_{4}-\mathrm{ACC}$ & 1.35 & 7.32 \\
\hline $\mathrm{GA}_{4} / \mathrm{d}_{2}-\mathrm{GA}_{4}$ & 0.24 & 7.11 \\
\hline $\mathrm{GA}_{9} / \mathrm{d}_{2}-\mathrm{GA}_{9}$ & 0.18 & 5.17 \\
\hline $\mathrm{GA}_{24} / \mathrm{d}_{2}-\mathrm{GA}_{24}$ & 0.23 & 2.56 \\
\hline
\end{tabular}

${ }^{a}$ Relative retention time

${ }^{\mathrm{b}}$ Relative peak area

old leaves of control plants is associated with a $75 \%$ reduction of the active cytokinin, zeatin (while other cytokinins are not significantly altered) and $40 \%$ or even higher reductions in $\mathrm{ABA}, \mathrm{JA}$ and $\mathrm{SA}$. It was found that this hormone profile (differences between old and young leaves) is completely altered in salt-stressed plants, in which neither cytokinin nor the anti-stress defense compounds ABA and SA decrease significantly in old leaves, however, JA levels remain constant. In other words, old leaves of salt-stressed plants showed higher levels of $\mathrm{Z}$, ABA, and SA than old leaves of control plants, although differences were significant for ABA only, with 8-fold higher levels in old leaves of salt-stressed compared to control plants. It appears from this hormone profile that reductions in $Z$ levels may trigger leaf senescence in control plants, increases in ABA are the responsible for triggering plant defense in both young and old leaves of salt-stressed plants. Obviously, this response will be affected by a cross-talk between different signaling pathways that need to be evaluated in depth using other complementary experimental approaches.

Special mention deserves the case of GAs, since more than 100 GAs are found in plants, although only a few of these are known to have biological activity such as $\mathrm{GA}_{4}$ [37]. Of the six analyzed gibberellins, only $\mathrm{GA}_{4}$, $\mathrm{GA}_{9}$ and $\mathrm{GA}_{24}$ were found in rosemary leaves. $\mathrm{GA}_{4}$

Table 5 Relative water content (RWC) and maximum efficiency of PSIl photochemistry $\left(F_{v} / F_{m}\right.$ ratio, indicative of damage to PSII) in Rosmarinus officinalis leaves of control and salt-stressed plants (treated with $200 \mathrm{mM}$ $\mathrm{NaCl}$ for 8 days).

\begin{tabular}{lcc}
\hline & $\begin{array}{c}\text { RWC } \\
(\%)\end{array}$ & $F_{v} / F_{m}$ \\
\hline Control young leaves & $80.83 \pm 1.63^{\mathrm{a}}$ & $0.837 \pm 0.02^{\mathrm{a}}$ \\
Control old leaves & $83.09 \pm 1.57^{\mathrm{a}}$ & $0.837 \pm 0.01^{\mathrm{a}}$ \\
Stress young leaves & $73.83 \pm 1.78^{\mathrm{b}}$ & $0.728 \pm 0.03^{\mathrm{b}}$ \\
Stress old leaves & $61.32 \pm 1.10^{\mathrm{c}}$ & $0.633 \pm 0.04^{\mathrm{b}}$ \\
\hline
\end{tabular}




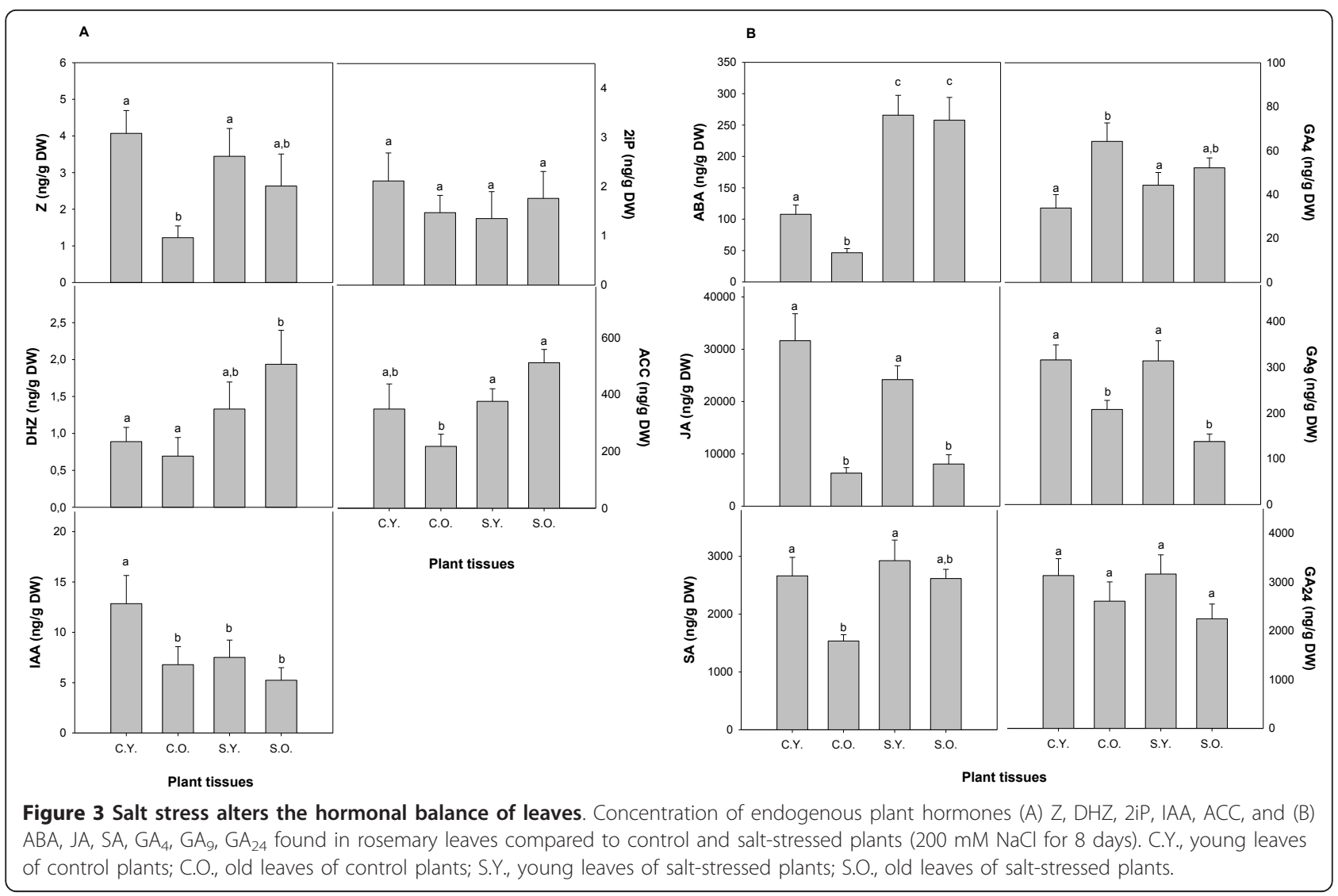

levels showed significant higher levels about $40 \%$ for old leaves in control plants compared to young leaves (Figure 3 ). Interestingly, significant higher levels of $\mathrm{GA}_{9}$, the immediate precursor of $\mathrm{GA}_{4}$, were observed for young leaves of both control and salt-stressed plants compared to old leaves, thus indicating a specific $\mathrm{GA}_{9}$ to $\mathrm{GA}_{4}$ conversion with the induction of leaf senescence in rosemary plants. However, it should be noted that $\mathrm{GA}_{4}$ levels were an order of magnitude lower than those of $\mathrm{GA}_{9}$, thus suggesting that the latter is precursor of different GAs.

To our knowledge we present the first hormone profile including ABA, ACC, auxins, cytokinins, GAs, JA and SA of rosemary plants in response to salinity comparing young and old leaves. In the literature ABA, IAA, ACC and cytokinin levels have been reported to be altered in $R$. officinalis leaves under water stress using ELISA and GC-MS/MS analyses (Table 6) [38,39]. While ABA and ACC levels were comparable to our results, auxin levels were 20 -fold higher than those obtained here. Furthermore, ZR was reported to occur in this species, while this cytokinin was not detected in the present study. In this previous study, the cross-reactivity of the antibodies used was as follows: ZR at $100 \%$ and $\mathrm{Z}$ at $88 \%$, which could explain at least partly the discrepancy between both studies. Aside from the differences in the experimental approaches used, plant and leaf developmental stages, life history traits, plant varieties and type, duration and magnitude of stress imposed on plants will also determine the differences in the levels of the different hormones. It is shown here, for instance, how salt stress and leaf age can significantly alter the hormone profile of leaves.

\section{Conclusions}

The development of a rapid, sensitive, high throughput, cost effective method for quantification of 17 endogenous plant hormones from seven plant classes in a complex matrix is described. The use of UPLC/ESI-MS/MS with multiple reaction monitoring (MRM) allowed each sample could be analyzed within 6 minutes. The method requires minimal plant tissue, is highly reproducible and was applicable to analyze dynamic changes in endogenous concentrations of hormones in plants exposed to salt stress. Due to the structural diversity of plant hormones no one extraction solvent was capable of extract all plant hormones equally well. If we aim at reducing extraction steps to a minimum, depending on the plant hormones of interest a different solvent is recommended: methanol:isopropanol:glacial acetic acid, 20:79:1 (v/v/v) for ABA, SA, JA, IAA and GAs, methanol:isopropanol:glacial acetic acid, 
Table 6 Comparing plant hormones contents from Rosmarinus officinalis leaves analyzed by different methods.

\begin{tabular}{lccc}
\hline & ELISA & GC-MS/MS & UPLC-MS/MS (this study) \\
\hline & {$[\mathbf{3 8}]$} & $(\mathrm{pmol39]})$ & $(\mathrm{pmol} / \mathrm{g} \mathrm{DW})$ \\
ABA & $(\mathrm{pmol} / \mathrm{g} \mathrm{FW})$ & $400-1500$ & $200-1000$ \\
ACC & - & $1000-16000$ & $2000-5000$ \\
IAA & - & $200-1500$ & $30-70$ \\
ZR equiv. & & - & - \\
$\begin{array}{l}\text { Experimental } \\
\text { conditions }\end{array}$ & Water-stressed plants under controlled & Field-grown plants exposed to summer & Salt-stressed plants under controlled \\
& conditions & drought & conditions \\
\hline
\end{tabular}

60:39:1 (v/v/v) for cytokinins, and methanol:glacial acetic acid, 99:1 (v/v) for ACC. Furthermore, this method is equally applicable to fresh or freeze dried tissue. Moreover, high loss of plant hormones during drying of sample extracts under $\mathrm{N}_{2}$ and re-suspension of the residues indicates that sample extracts should immediately be injected. However, the adjustment of sample weight and volume of the extraction solvent must be taken into account. As a summary, a schematic extraction diagram to analyze small amounts of plant material without time-consuming additional steps such as purification, sample drying under $\mathrm{N}_{2}$ and re-suspension of the residues using UPLC/ESI-MS/ MS with MRM is shown in Figure 4. This method has been used in our group to obtain the hormone profile of a

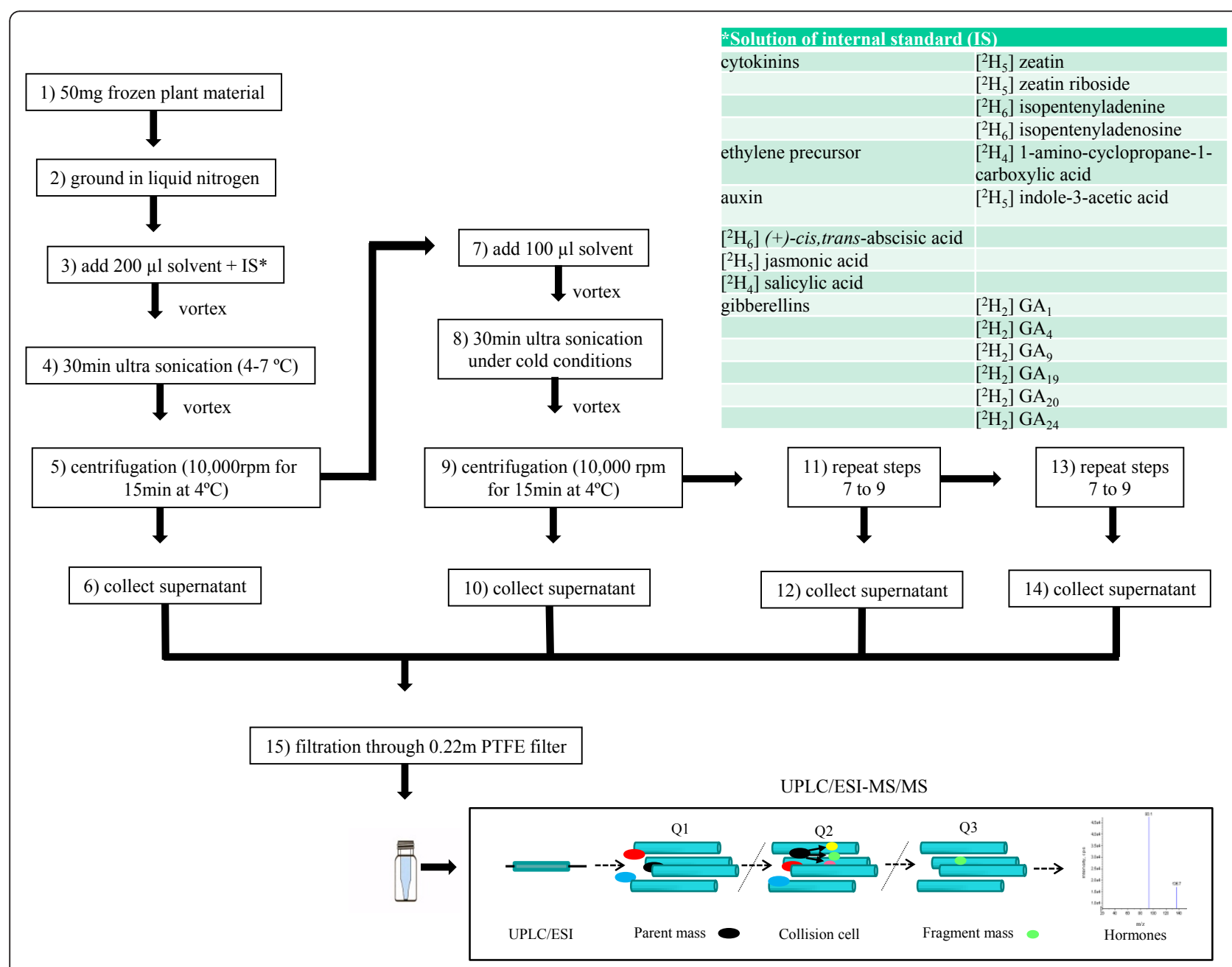

Figure 4 Summary of the extraction protocol and analysis. Schematic extraction diagram to analyze small amounts of plant material using UPLC/ESI-MS/MS with multiple reaction monitoring (MRM). 
number of plant tissues, including leaves, flowers and seeds of different species of a number of plant families (including the model plant Arabidopsis thaliana and other Brassicaceae, Liliaceae, Cistaceae, Anacardiaceae, Dioscoreaceae, Velloziaceae, Xyridaceae and Lamiaceae) that all bring completely different matrices and therefore their specific complexity for analyses. As shown in the present study, each species will require a specific handling during extraction and analysis, and the schematic diagram presented in Figure 4 can serve as a basis to the optimization of the method for each species. Although obtaining the hormone profile of species with complex matrices may be more difficult due to the presence of possible interfering compounds, it is also challenging and will undoubtedly be needed in the near future if we are to better understand plant stress responses in species of contrasting habitats that can be used as model plants for the study of plant responses to environmental stresses.

\section{Methods}

\section{Chemicals}

Unlabeled ACC, IPA, Z, ZR, IAA, ABA, JA, SA were purchased from Sigma-Aldrich (Steinheim, Germany). Unlabeled 2iP, DHZ, DHZR, and deuterium labeled $\mathrm{d}_{4}$ ACC, $d_{6}-$ IPA, $d_{5}-Z, d_{5}-Z R, d_{5}-$ IAA, $d_{6}-A B A, d_{4}-S A, d_{5}-$ $J A$ and $d_{6}-2 i P$ were purchased from OlChemim Ltd. (Olomouc, Czech Republic). Unlabeled and deuterium labeled gibberellins $\mathrm{GA}_{1}, \mathrm{GA}_{4}, \mathrm{GA}_{9}, \mathrm{GA}_{19}, \mathrm{GA}_{20}, \mathrm{GA}_{24}$, $\mathrm{d}_{2}-\mathrm{GA}_{1}, \mathrm{~d}_{2}-\mathrm{GA}_{4}, \mathrm{~d}_{2}-\mathrm{GA}_{9}, \mathrm{~d}_{2}-\mathrm{GA}_{19}, \mathrm{~d}_{2}-\mathrm{GA}_{20}, \mathrm{~d}_{2}-\mathrm{GA}_{24}$ were purchased from Dr. Lewis Mander at the Australian National University (Canberra, Australia).

\section{Plant material and sampling}

All work was carried out with rosemary leaves. Fifteen plants were purchased from a nursery (Vic, Spain) and maintained in a greenhouse at the experimental fields of the University of Barcelona (Barcelona, Spain) with controlled temperature $\left(24 / 18^{\circ} \mathrm{C}\right)$ and adequate water conditions by irrigating the plants with half concentration of Hoagland solution every 2 days. For the optimization procedure of sample preparation and extraction, leaves from these plants were collected, frozen in liquid nitrogen and stored at $-80^{\circ} \mathrm{C}$ until analysis. For the salinity experiment, five plants were exposed to salt stress by irrigating with the same nutrient solution containing an extra addition of 200 $\mathrm{mM} \mathrm{NaCl}$, and compared to five control plants (all plants were watered every 2 days). Young and old leaves from the uppermost and lowest part of the plant, respectively, were collected after 8 days of treatment, frozen in liquid nitrogen and stored at $-80^{\circ} \mathrm{C}$ until analyses.

\section{Leaf water content and chlorophyll fluorescence}

To determine the relative water content (RWC) of leaf material from the salinity experiment, young and old leaves were collected, immediately weighed (FW), re-hydrated for $24 \mathrm{~h}$ at $4^{\circ} \mathrm{C}$ in darkness (TW) and subsequently oven-dried for $48 \mathrm{~h}$ at $60^{\circ} \mathrm{C}(\mathrm{DW})$. The RWC was determined as $100 \times$ (FW-DW)/(TW-DW). Measurements of the maximum efficiency of photosystem II photochemistry $\left(\mathrm{F}_{\mathrm{v}} / \mathrm{F}_{\mathrm{m}}\right.$ ratio $)$ were made by using a pulse-modulated fluorimeter Imaging-PAM (Walz, Effeltrich, Germany) after $2 \mathrm{~h}$ of dark adaptation. The $F_{v} / F_{m}$ ratio was calculated as $\left(\mathrm{F}_{\mathrm{m}}-\mathrm{F}_{0}\right) / \mathrm{Fm}$, where $F_{m}$ and $F_{0}$ are the maximum and basal fluorescence yields, respectively, of dark-adapted leaves.

\section{Sample preparation}

Frozen leaf material (about $100 \mathrm{mg}$ FW.) was ground in liquid nitrogen with the mixer mill MM400 (Retsch $\mathrm{GmbH}$, Haan, Germany) in a $2 \mathrm{ml}$ Eppendorf tube, and then extracted with $1 \mathrm{ml}$ of extraction solvent (methanol:isopropanol, 20:80 (v/v) with 1\% of glacial acetic acid) using ultra sonication $\left(4-7^{\circ} \mathrm{C}\right)$. The labeled forms of the compounds $\mathrm{d}_{4}-\mathrm{SA}, \mathrm{d}_{6}-\mathrm{ABA}, \mathrm{d}_{5}-\mathrm{JA}, \mathrm{d}_{5}-\mathrm{IAA}, \mathrm{d}_{2^{-}}$ $\mathrm{GA}_{1}, \mathrm{~d}_{2}-\mathrm{GA}_{4}, \mathrm{~d}_{2}-\mathrm{GA}_{9}, \mathrm{~d}_{2}-\mathrm{GA}_{19}, \mathrm{~d}_{2}-\mathrm{GA}_{20}, \mathrm{~d}_{2}-\mathrm{GA}_{24}, \mathrm{~d}_{4}-$ ACC, $d_{6}-2 i P, d_{6}-$ IPA, $d_{5}-Z$ and $d_{5}-Z R$ were added as internal standards. $D_{5}-Z$ and $d_{5}-Z R$ were used as internal standards for DHZ and DHZR, respectively. After centrifugation $\left(10,000 \mathrm{rpm}\right.$ for $15 \mathrm{~min}$ at $\left.4^{\circ} \mathrm{C}\right)$, the supernatant was collected and the pellet was reextracted with $0.5 \mathrm{ml}$ of extraction solvent and the extraction repeated three times again. Then, supernatants were combined and dried completely under a nitrogen stream and re-dissolved in $300 \mu \mathrm{l}$ of methanol, centrifuged (10,000 rpm for $5 \mathrm{~min})$ and filtered through a $0.22 \mu \mathrm{m}$ PTFE filter (Waters, Milford, MA, USA). Samples $(5 \mu \mathrm{l})$ were then analyzed by UPLC/ESI-MS/ MS. Hormones were determined in ten independent samples for each treatment. Quantification was done by the creation of calibration curves including each of the 17 unlabeled analyte compounds (SA, ABA, JA, IAA, GA1, GA $4, \mathrm{GA}_{9}, \mathrm{GA}_{19}, \mathrm{GA}_{20}, \mathrm{GA}_{24}, \mathrm{ACC}, 2 \mathrm{P}, \mathrm{IPA}, \mathrm{Z}$, $\mathrm{ZR}, \mathrm{DHZ}$ and DHZR). Ten standard solutions were prepared ranging from 0.05 to $1000 \mathrm{ng} \mathrm{ml}^{-1}$ and for each solution a constant amount of internal standard (as described above) was added. Calibration curves for each analyte were generated using Analyst ${ }^{\mathrm{TM}}$ software (Applied Biosystems, Inc., California, USA). The limit of detection (LOD, $\mathrm{S} / \mathrm{N}=3$ ) and the limit of quantification (LOQ, $/ \mathrm{N}=10$ ) were also calculated with the aid of this software.

\section{UPLC/ESI-qMS/MS analysis}

The UPLC system consisted of an Aquity UPLCTM System (Waters, Milford, MA USA) quaternary pump equipped with an autosampler. For the analysis of the extracts, a HALO ${ }^{\mathrm{TM}} \mathrm{C} 18$ (Advanced Materials Technology, Inc., Wilmington, USA) column $(2.1 \times 75 \mathrm{~mm}, 2.7$ $\mu \mathrm{m})$ was used. Gradient elution was done with water 
and $0.05 \%$ glacial acetic acid (solvent A) and acetonitrile with $0.05 \%$ glacial acetic acid (solvent B) at a constant flow rate of $0.6 \mathrm{ml} \mathrm{min}^{-1}$. Cytokinins and ACC were analyzed using method 1 (M1) and ABA, JA, SA, IAA, and gibberellins were analyzed using method 2 (M2). The gradient profile for M1 (cytokinins and ACC) was applied as follow ( $t$ (min), \% A): (0, 99), (2, 0), (2.40, 0), (2.60, 99), (3, 99). The gradient profile for M2 (ABA, JA, SA, IAA, and gibberellins) was applied as follow: $(t(\mathrm{~min}), \% \mathrm{~A}):(0,99),(2.20,0)$, (2.40, 0), (2.60, 99), (3, 99). MS and MS/MS experiments were performed on an API 3000 triple quadrupole mass spectrometer (PE Sciex, Concord, Ont., Canada). Analyses for M1 were performed using Turbo Ionspray source in positive ion mode and for $\mathrm{M} 2$ in negative ion mode. For both methods temperature was $400^{\circ} \mathrm{C}$, nebulizer gas $\left(\mathrm{N}_{2}\right)$ 10 (arbitrary units), curtain gas $\left(\mathrm{N}_{2}\right) 12$ (arbitrary units), collision gas $\left(\mathrm{N}_{2}\right) 4$ (arbitrary units) and the capillary voltage was $3.5 \mathrm{kV}$ for M1 and $-3.5 \mathrm{kV}$ for M2, respectively. The optimized MS/MS conditions for the analysis of plant hormones are summarized in Additional File 4 and were determined in infusion experiments: a standard solution of each plant hormone and deuterium labeled plant hormone was infused of a constant flow rate of $15 \mu \mathrm{min}^{-1}$ into the mass spectrometer using a Model 11 syringe pump (Harvard Apparatus, Holliston, MA, USA). The mass spectrometer was operated in multiple reaction mode (MRM) due to their high selectivity using precursor-to-product ion transitions because many compounds could present the same nominal molecular mass or peaks can overlap. Since more than 100 GAs with partly same molecular masses and similar retention times are found in plants special mention is needed for GAs identification in plant extracts. Additional files 5 and 6 show fragmentation patterns for labeled and unlabeled $\mathrm{GA}_{1}, \mathrm{GA}_{4}, \mathrm{GA}_{9}, \mathrm{GA}_{19}, \mathrm{GA}_{20}, \mathrm{GA}_{24}$ standards. In rosemary leaf extracts $\mathrm{GA}_{4}, \mathrm{GA}_{9}$ and $\mathrm{GA}_{24}$ were detected. Using multiple reaction monitoring (MRM) conditions a specific precursor to one product ion transition is monitored. However, to verify the identification of GAs in rosemary leaf extracts the specific precursor ions of $\mathrm{GA}_{4}, \mathrm{GA}_{9}$ and $\mathrm{GA}_{24}$ to two different product ions in MRM mode were monitored. All GAs mass chromatograms from rosemary leaf extracts showed identical retention times as GA standards.

\section{Statistical analyses}

Differences between treatments were evaluated using the analysis of variance (ANOVA), using the DMS's post hoc test, and were considered significant at a probability level of $\mathrm{P}<0.05$.

\section{Additional material}

Additional file 1: Effects of freeze-drying. Concentration of endogenous plant hormones (A) Z, DHZ, 2iP, IAA, ACC, and (B) ABA, JA,
$\mathrm{SA}_{1}, \mathrm{GA}_{4}, \mathrm{GA}_{9}, \mathrm{GA}_{24}$ detected in fresh weight (FW) and freeze-dried (DW) rosemary leaves.

Additional file 2: Extraction efficiency. Concentration of (A) Z, DHZ, 2iP, IAA, ACC, and (B) ABA, JA, SA, GA $4, G_{9}, G_{24}$ found in rosemary leaves after 1,2, 3, 4 and 5 extraction procedures.

Additional file 3: Effects of the amount of plant material used for extraction. Capacity of extraction method to analyze different leaf amounts (20 - $200 \mathrm{mg} \mathrm{FW)} \mathrm{of} \mathrm{rosemary} \mathrm{leaves.}$

Additional file 4: Optimized UPLC/ESI-MS/MS parameters. Parameters are listed in multiple reaction monitoring (MRM) conditions for quantification of plant hormones.

Additional file 5: Fragmentation patterns of labeled and unlabeled $\mathrm{GA}_{1}, \mathrm{GA}_{19}$, and $\mathrm{GA}_{20}$ standards. (A) $\mathrm{GA}_{1}$ (precursor $\mathrm{m} / \mathrm{z} 347$ and product $\mathrm{m} / \mathrm{z} 273$ ions) and $\mathrm{d}_{2}-\mathrm{GA}_{1}$ standards (precursor $\mathrm{m} / \mathrm{z} 349$ and product $\mathrm{m} / \mathrm{z} 275$ ions). (B) $\mathrm{GA}_{19}$ (precursor $\mathrm{m} / \mathrm{z} 361$ and product $\mathrm{m} / \mathrm{z} 273$ ions) and $\mathrm{d}_{2}-\mathrm{GA}_{19}$ standards (precursor $\mathrm{m} / \mathrm{z} 363$ and product $\mathrm{m} / \mathrm{z} 275$ ions), (C) $\mathrm{GA}_{20}$ (precursor $\mathrm{m} / \mathrm{z} 331$ and product $\mathrm{m} / \mathrm{z} 287$ ions) and $\mathrm{d}_{2^{-}}$ $\mathrm{GA}_{20}$ standards (precursor $\mathrm{m} / \mathrm{z} 333$ and product $\mathrm{m} / \mathrm{z} 289$ ions)

Additional file 6: Fragmentation patterns of labeled and unlabeled $\mathrm{GA}_{4}, \mathrm{GA}_{9}, \mathrm{GA}_{24}$ standards and identification of $\mathrm{GA}_{4}, \mathrm{GA}_{9}$ and $\mathrm{GA}_{24}$ in Rosmarinus officinalis extracts. (A1) Fragmentation patterns of $\mathrm{GA}_{4}$ (precursor $\mathrm{m} / \mathrm{z} 331$ and product $\mathrm{m} / \mathrm{z} 213$ ions) and $\mathrm{d}_{2}-\mathrm{GA}_{4}$ standards (precursor $\mathrm{m} / \mathrm{z} 333$ and product m/z 215 ions). (A2) UPLC/ESI-MS/MS chromatograms from rosemary leaf extract using multiple reaction monitoring (MRM) conditions. $\mathrm{GA}_{4}$ was identified by monitoring the precursor ion $(\mathrm{m} / \mathrm{z} 331)$ to two different product ion transitions $(\mathrm{m} / \mathrm{z} 213$ and $m / z 225$ ) in MRM mode with identical retention times as the GA standard. (B1) Fragmentation patterns of $\mathrm{GA}_{9}$ (precursor $\mathrm{m} / \mathrm{z} 315$ and product $\mathrm{m} / \mathrm{z} 271$ ions) and $\mathrm{d}_{2}-\mathrm{GA}_{9}$ standards (precursor $\mathrm{m} / \mathrm{z} 317$ and product $\mathrm{m} / \mathrm{z} 273$ ions). (B2) UPLC/ESI-MS/MS chromatograms from rosemary leaf extract using multiple reaction monitoring (MRM) conditions. $G A_{9}$ was identified by monitoring the precursor ion $(\mathrm{m} / \mathrm{z} 315)$ to two different product ion transitions ( $m / z 271$ and $m / z 253$ ) in MRM mode with identical retention times as the $\mathrm{GA}_{9}$ standard. (C1) Fragmentation patterns of $\mathrm{GA}_{24}$ (precursor $\mathrm{m} / \mathrm{z} 345$ and product m/z 257 ions) and $\mathrm{d}_{2}-\mathrm{GA}_{24}$ standards (precursor $\mathrm{m} / \mathrm{z} 347$ and product $\mathrm{m} / \mathrm{z} 259$ ions). (C2) UPLC/ESI-MS/MS chromatograms from rosemary leaf extract using multiple reaction monitoring (MRM) conditions. $\mathrm{GA}_{24}$ was identified by monitoring the precursor ion $(\mathrm{m} / \mathrm{z} 345)$ to two different product ion transitions $(\mathrm{m} / \mathrm{z} 257$ and $\mathrm{m} / \mathrm{z}$ 301) in MRM mode with identical retention times as the $\mathrm{GA}_{24}$ standard.

\section{Abbreviations}

ABA: abscisic acid; ACC: 1-amino-cyclopropane-1-carboxyic acid; CE: collision energy: CXP: collision cell exit potential; DHZ: dihydrozeatin; DHZR: dihydrozeatin riboside; DP: declustering potencial; EP: entrance potential; ESIMS/MS: electrospray ionization tandem mass spectrometry; FP: focusing potential; GA: gibberellin; IAA: índole-3-acetic acid; 2iP: isopentenyladenine: IPA: isopentenyladenosine; JA: jasmonic acid; LOD: limit of detection; LOQ: limit of quantification; MRM: multiple reaction monitoring; OPDA: 12-oxophytodienoic acid; RDS: relative deviation standard; SA: salicylic acid; UPLC: ultrahigh performance liquid chromatography; Z: zeatin; ZR: zeatin riboside.

\section{Acknowledgements}

Support for the research was received through grants BFU2006-01127, BFU2009-07294-E, BFU2009-06045, and CSD2008-00040 from the Ministry of Science and Innovation of the Spanish Government, and the ICREA

Academia prize to SMB funded by the Generalitat de Catalunya. The authors wish to thank all lab members (M.E. Abreu, I. Hernández, M.A. Asensi-Fabado, J. Cela, M. Oñate and L. Arrom) for their help in method optimization during the last 5 years. We are also especially indebted to the technical staff of the Serveis Científico-Tècnics of the University of Barcelona, and particularly $\mathrm{O}$. Jáuregui for their help in method optimization.

\section{Authors' contributions}

MM participated in the design of the study, carried out the lab work and helped to draft the manuscript. SMB conceived of the study, and 
participated in its design and coordination and helped to draft the manuscript. All authors have read and approved the final manuscript.

\section{Competing interests}

The authors declare that they have no competing interests.

Received: 12 July 2011 Accepted: 18 November 2011 Published: 18 November 2011

\section{References}

1. Pan $X$, Welti $R$, Wang $X$ : Simultaneous quantification of major phytohormones and related compounds in crude plant extracts by liquid chromatography tandem mass spectrometry. Phytochemistry 2008, 69:1773-1781.

2. Dun EA, Brewer PB, Beveridge CA: Strigolactones: discovery of the elusive shoot branching hormone. Trends Plant Sci 2009, 14:364-372.

3. Davies PJ: The plant hormones: their nature, occurrence, and functions. Kluwer Academic Publishers, Netherlands; 2010.

4. Ross JJ, O'Neill DP, Rathbone DA: Auxin-gibberellin interactions in pea: integrating the old with the new. J Plant Growth Regul 2003, 22:99-108.

5. Tanaka M, Takei K, Kojima M, Sakakibara H, Mori H: Auxin controls local cytokinin biosynthesis in the nodal stem in apical dominance. Plant $J$ 2006, 45:1028-1036.

6. Goda H, Sawa S, Asami T, Fujioka S, Shimada Y, Yoshida S: Comprehensive comparison of auxin-regulated and brassinosteroid-regulated genes in Arabidopsis. Plant Physiol 2004, 134:1555-1573.

7. Nemhauser JL, Mockler TC, Chory J: Interdependency of brassinosteroid and auxin signaling in Arabidopsis. PLOS Biol 2004, 2:E258.

8. Cary AJ, Liu W, Howell SH: Cytokinin action is coupled to ethylene in its effects on the inhibition of root and hypocotyl elongation in Arabidopsis thaliana seedlings. Plant Physiol 1995, 107:1075-1082.

9. Gazzarrini S, McCourt P: Genetic interactions between ABA, ethylene and sugar signaling pathways. Curr Opin Plant Biol 2001, 4:387-391.

10. Wilkinson S, Davies WJ: Drought, ozone, ABA and ethylene: new insights from cell to plant community. Plant Cell Environ 2010, 33:510-525.

11. Wang D, Pajeroska-Mukhtar K, Hendrickson Culler A, Dong X: Salicylic acid inhibits pathogen growth in plants trough repression of the auxin signalling pathway. Curr Biol 2007, 17:1784-90

12. Vlot AC, Amick Dempsey D'M, Klessing DF: Salicylic acid, a multifaceted hormone to combat disease. Annu Rev Phytopathol 2009, 47:177-206.

13. Müller A, Duchting P, Weiler EW: A multiplex GC-MS/MS technique for the sensitive and quantitative single-run analysis of acidic phytohormones and related compounds, and its application to Arabidopsis thaliana. Planta 2002, 216:44-56.

14. Pan $X$, Wang $X$ : Profiling of plant hormones by mass spectrometry. J Chromatogr B 2009, 877:2806-2813.

15. Chiwocha SD, Abrams SR, Ambrose SJ, Cutler AJ, Loewen M, Ross AR, Kermode AR: A method for profiling classes of plant hormones and their metabolites using liquid chromatography-electrospray ionization tandem mass spectrometry: an analysis of hormone regulation of thermodormancy of lettuce (Lactuca sativa L.) seeds. Plant J 2003, 35:405-417.

16. Kojima M, Kamada-Nobusada T, Komatsu H, Takei K, Kuroha T, Mizutani M Ashikari M, Ueguchi-Tanaka M, Matsuoka M, Suzuki K, Sakakibara H: Highly sensitive and high-throuput analysis of plant hormones using MS-probe modification and liquid chromatography-tandem mass spectrometry: an application for hormone profiling in Oryza sativa. Plant Cell Physiol 2009, 50:1201-1214.

17. Izumi Y, Okazawa A, Bamba T, Kobayashi A, Fukusaki E: Development of a method for comprehensive and quantitative analysis of plant hormones by highly sensitive nanoflow liquid chromatography-electrospray ionization-ion trap mass spectrometry. Anal Chim Acta 2009, 649:215-225.

18. Matsuura H, Aoi A, Satou C, Nakaya M, Masuta C, Nabeta K: Simultaneous UPLC MS/MS analysis of endogenous jasmonic acid, salicylic acid and their related compounds. Plant Growth Regul 2009, 57:293-301.

19. Chen W, Gai Y, Liu S, Wang R, Jiang X: Quantitative analysis of cytokinins in plants by high performance liquid chromatography: electronspray ionization ion trap mass spectrometry. J Integr Plant Biol 2010, 52:925-932.

20. Segarra G, Jauregui O, Casanova E, Trillas I: Simultaneous quantitative LCESI-MS/MS analyses of salicylic acid and jasmonic acid in crude extracts of Cucumis sativus under biotic stress. Phytochemistry 2006, 67:395-401.
21. Volkmar KM, Hu Y, Steppuhn H: Physiological responses of plants to salinity: a review. Can J Plant Sci 1998, 78:19-27.

22. Ghanem ME, Albacete A, Martínez-Andújar C, Acosta M, Romero-Aranda R, Dodd IA, Lutts S, Pérez-Alfocea F: Hormonal changes during salinityinduced leaf senescence in tomato (Solanum lycopersicum L.). J Exp Bot 2008, 59:3039-3050.

23. Khan MN, Siddiqui MH, Mohammad F, Naeem M, Khan MMA: Calcium chloride and gibberellic acid protect linseed (Linum usitatissimum L.) from $\mathrm{NaCl}$ stress by inducing antioxidative defence system and osmoprotectant accumulation. Acta Physiol Plant 2010, 32:121-132.

24. Pic E, Teyssendier B, Tardieu F, Ture O: Leaf senescence induced by mild water deficit follows the same sequence of macroscopic, biochemical, and molecular events as monocarpic senescence in Pea1. Plant Physiol 2002, 128:236-246.

25. Leung J, Giraudat J: Abscisic acid signal transduction. Annu Rev Physiol Plant Mol Biol 1998, 49:199-222

26. Netting AG: pH, abscisic acid and the integration of metabolism in plants under stressed and non-stressed conditions: cellular responses to stress and their implication for plant water relations. J Exp Bot 2000, 51:147-158.

27. Forcat S, Bennett MH, Mansfield JW, Grant MR: A rapid and robust method for simultaneously measuring changes in the phytohormones ABA, JA and SA in plants following biotic and abiotic stress. Plant Meth 2008, 4:16-23.

28. Miyamoto S, Martinez I, Podilla M, Portillo A, Ornelas D: Landscape plant lists of salt tolerance assessment. Texas \& University Agricultural Research and Extension Center, El Paso; 2004

29. Tounekti T, Vadel AM, Oñate M, Khemira H, Munné-Bosch S: Salt-induced oxidative stress in rosemary plants: Damage or protection? Environ Exp Bot 2011, 71:298-305.

30. Cramer GR: Response of abscisic acid mutants of Arabidopsis to salinity. Funct Plant Biol 2002, 29:561-567.

31. Raghavendra AS, Gonugunta VK, Christmann A, Grill E: ABA perception and signaling. Trends Plant Sci 2010, 15:395-401.

32. Munns R: Comparative physiology of salt and water stress. Plant Cell Environ 2000, 25:239-250

33. Wi SJ, Park KY: Antisense expression of carnation cDNA encoding ACC synthase or ACC oxidase enhances polyamine content and abiotic stress tolerance in transgenic tobacco plants. Mol Cell 2002, 13:209-222.

34. Li L, Zhao Y, McCaig BC, Wingerd BA, Wang J, Whalon ME, Pichersky E, Howe GA: The tomato homolog of CORONATINE-INSENSITIVE1 is required for the maternal control of seed maturation, jasmonatesignaled defense responses, and glandular trichome development. Plant Cell 2004, 16:126-143.

35. Devoto A, Turner JG: Jasmonate-regulated Arabidopsis stress signaling network. Physiol Plant 2005, 123:161-172.

36. Najafian S, Khoshkhui M, Tavallali V: Effect of salicylic acid and salinity in rosemary (Rosmarinus officinalis L.): Investigation on changes in gas exchange, water relations, and membrane stabilization. Adv Environ Biol 2009, 3:322-328.

37. Richards DE, King KE, Ait-ali T, Harberd NP: How gibberellin regulates plant growth and development: a molecular genetic analysis of gibberellin signalling. Annu Rev Plant Biol 2001, 52:67-88.

38. López-Carbonell M, Alegre L, Pastor A, Prinsen E, van Onckelen H: Variation in abscisic acid, indole-3-acetic acid and zeatin riboside concentations in two Mediterranean shrubs subjected to water stress. Plant Growth Regul 1996, 20:271-277.

39. Munné-Bosch S, López-Carbonell M, Alegre L, van Onckelen HA: Effect of drought and high solar radiation on 1-aminocyclopropane-1-carboxylic acid and abscisic acid concentrations in Rosmarinus officinalis plants. Physiol Plant 2002, 114:380-386.

doi:10.1186/1746-4811-7-37

Cite this article as: Müller and Munné-Bosch: Rapid and sensitive hormonal profiling of complex plant samples by liquid chromatography coupled to electrospray ionization tandem mass spectrometry. Plant Methods 2011 7:37. 